\title{
Intra-arterial injection of diazepam
}

\author{
Sean Rice BA MSc, Ronald H Levine MD CM FRCSC \\ St Joseph's Health Centre, Toronto, Ontario
}

\begin{abstract}
S Rice, RH Levine. Intra-arterial injection of diazepam. Can J Plast Surg 1996;4(2):121-122. Diazepam is frequently and safely used in a wide variety of medical settings. However, an accidental intra-arterial injection of diazepam can have serious sequelae. This report describes the case of a intra-arterial injection of diazepam and outlines the current suggested treatment.
\end{abstract}

Key Words: Diazepam, Intra-arterial injection

\section{Injection intra-artérielle de diazépam}

RÉSUMÉ : Le diazépam est souvent utilisé sans danger dans de nombreux contextes médicaux, mais l'injection intra-artérielle accidentelle de diazépam peut avoir des conséquences graves. Ce rapport décrit le cas d'un sujet ayant reçu une injection intraartérielle de diazépam et résume les mesures thérapeutiques suggérées à l'heure actuelle.

Intravenous diazepam is widely accepted as a safe treatment for patients in a convulsive state, or as a sedative preoperatively for minor surgery. However, an accidental intra-arterial injection of diazepam can be associated with serious sequelae, with reports ranging from short term pain (1) to extensive tissue necrosis and above elbow amputation (2). The following report describes the case of a young woman who received an accidental intra-arterial injection of diazepam into her radial artery before a cystoscopic examination, and outlines the current recommended treatment.

\section{CASE REPORT}

A 23-year-old female received a $10 \mathrm{mg}$ injection of diazepam into her right antecubital fossa before a cystoscopic examination. Upon injection of the diazepam the patient complained of severe burning pain in the forearm, and the urologist noticed immediate blanching of the skin distal to the injection site followed by swelling. Post cystoscopy the blanching and extreme pain persisted, and it was questioned as to whether this was an intra-arterial injection of diazepam. Examination revealed a significantly swollen forearm. There was a full active and passive range of motion of both the fingers and wrist; however, these movements were associated with moderate pain. Neurological status was normal, and a radial pulse was palpable. The patient was admitted overnight to the hospital with her arm elevated and icepacks applied regularly to control swelling. The next day she improved and was discharged home. However, two days later she returned to the emergency department with severe pain, swelling and slight mottling of the anterior forearm. Seven days post injection (Figure 1) the patient now had moderate swelling of the forearm, with increased mottling of the skin over the volar/radial aspect. 
Both active and passive range of motion was associated with intense pain in the fingers and forearm. A good radial pulse was palpable and neurological assessment was normal; there were no signs of infection or tissue necrosis. At this time the patient was started on oral prednisone, as well as aggressive physiotherapy to encourage movement of the limb. Four weeks post intra-arterial injection of diazepam the swelling of the arm and mottling of the skin subsided. Physical examination is now normal with pain free movement of the arm. No permanent damage appears to have resulted.

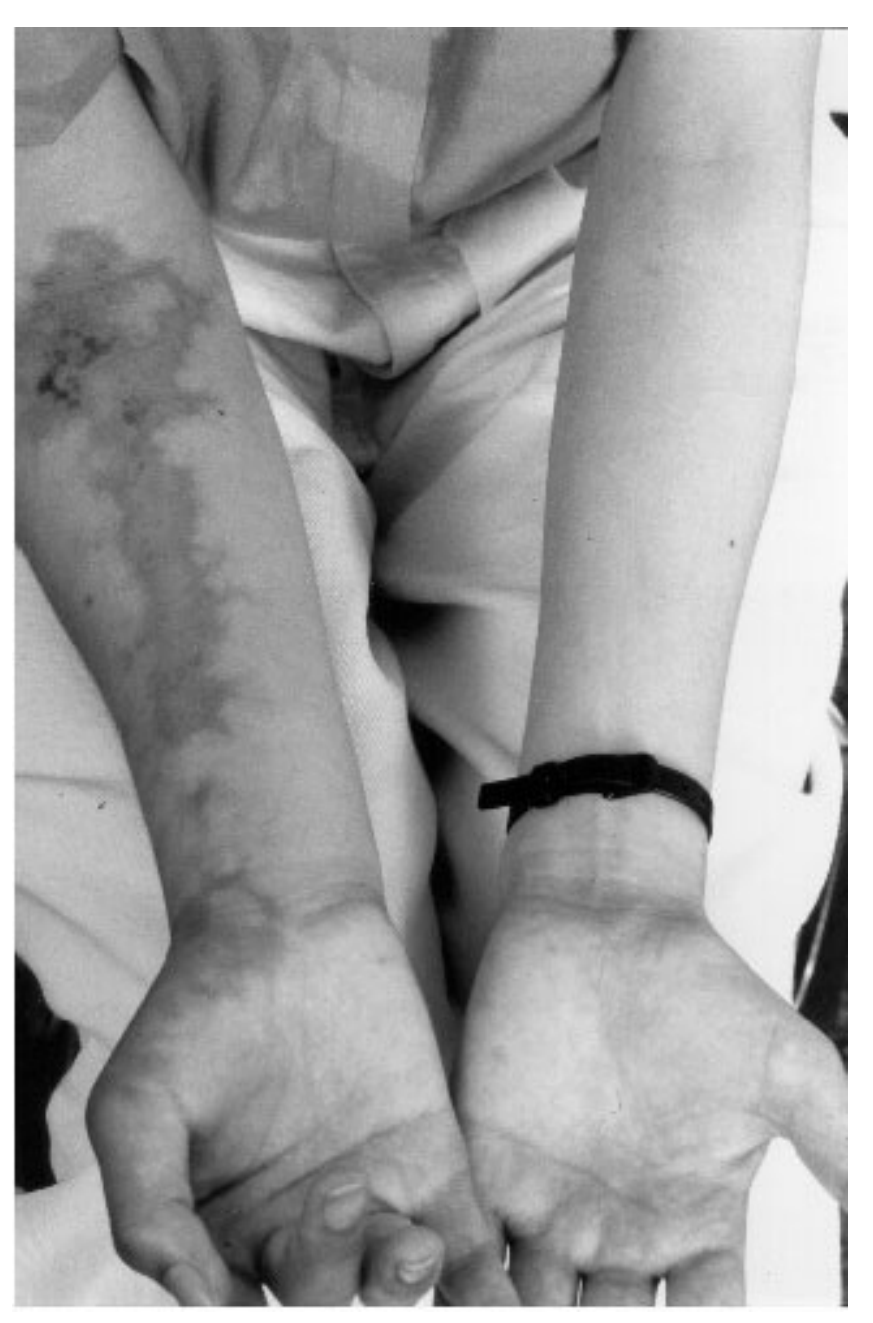

Figure 1) Mottling of skin seven days post injection

\section{DISCUSSION}

Case reports of intra-arterial injection of diazepam are rare; however, the outcome can be disastrous with loss of digits or limb (1-3). The sequence of events post injection are similar in most cases. Initially there is immediate intense pain and blanching of the skin distal to the injection site. The forearm quickly becomes edematous, however pulses 
and sensation are usually preserved. Mottling of the skin appears within hours to days and tracks along the outline of the affected artery. Skin necrosis usually appears between one and four weeks (1-3).

Diazepam is lipid soluble, containing benzyl alcohol, sodium benzoate and benzoic acid as suspension agents. These elements, in combination, are thought to have a dose related lytic effect on the endothelial walls of both arteries and capillaries (4). The initial response is immediate vasoconstriction followed by disruption of the endothelial membrane, which allows the intra-vascular contents to extrude causing edema. The damaged endothelial wall then allows platelet adherence and multiple thrombus occurrence which in turn occludes the arteries $(2,5)$.

Course of treatment depends on the time frame between the intra-arterial injection of diazepam and interaction with the patient. Upon injection of diazepam if the patient complains of severe immediate pain, and if one suspects an intra-arterial injection, stop immediately. Leave the needle in place and inject isotonic saline (3) or a vasodilator such as bupivacaine (5). This will both dilute the concentration of diazepam and increase blood flow in an attempt to flush out the noxious substances. An immediate intra-arterial injection of methylprednisolone has also been shown protect the endothelial wall (5).

Usually these patients present to the plastic surgeon sometime after the initial injection. The treatment at this point is symptomatic including; elevation, pain relief, use of anti-coagulants, physiotherapy and, if necessary, debridement of necrotic tissue (1-5).

Diazepam is one of the most commonly used drugs worldwide. An accidental intra-arterial injection of diazepam is a fairly uncommon occurrence, yet can be associated with a disastrous outcome.

\section{REFERENCES}

1. Schulenberg CE, et al. Intra-arterial diazepam. S Afr Med J 1985;68:891-2.

2. Schneider S, Mace J. Loss of limb following intravenous diazepam. Pediatrics 1974;53:112-4.

3. Ruddel $\mathrm{H}$, et al. Acute arterial occlusions due to accidental intra-arterial injections of diazepam. Klinis Wochensch 1985;63:93-4.

4. Gaspar M. Vascular Emergencies. New York: Appleton Press, 1982:241-50.

5. Knill RL, Evans D. Pathogenesis of gangrene following intra-arterial injection of drugs: a new hypothesis. Can Anaesth Soc J 1975;22:637-45. 